Y. Kitaoka

Nagoya Math. J.

Vol. 82 (1981), 99-111

\title{
TENSOR PRODUCTS OF POSITIVE DEFINITE QUADRATIC FORMS, V
}

\author{
YOSHIYUKI KITAOKA
}

Our aim is to prove

THeOREM. Let $L$ be a positive lattice of E-type such that $[L: \tilde{L}]<\infty$ and $\tilde{L}$ is indecomposable.

(i) If $L \cong L_{1} \otimes L_{2}$ for positive lattices $L_{1}, L_{2}$, then $L_{1}, L_{2}$ are of $E$-type and $\left[L_{1}: \tilde{L}_{1}\right],\left[L_{2}: \tilde{L}_{2}\right]<\infty$ and $\tilde{L}_{1}, \tilde{L}_{2}$ are indecomposable.

(ii) If $L$ is indecomposable with respect to tensor product, then for each indecomposable positive lattice $X$ we have

(1) $L \otimes X \cong L \otimes Y$ implies $X \cong Y$ for a positive lattice $Y$,

(2) If $X=\otimes^{t} L \otimes X^{\prime}$ where $X^{\prime}$ is not divided by $L$, then $O(L \otimes X)$ is generated by $O(L), O\left(X^{\prime}\right)$ and interchanges of $L$ 's, and

(3) $L \otimes X$ is indecomposable.

We must explain notations and terminology. By a positive lattice we mean a lattice on positive definite quadratic space over the rational number field $\boldsymbol{Q}$. Let $L$ be a positive lattice and put

$$
m(L)=\min _{\substack{x \in L \\ x \neq 0}} Q(x)
$$

where $Q(\quad)$ is a quadratic form associated with $L$. Set $\mathfrak{M}(L)=$ $\{x \in L \mid Q(x)=m(L)\} . \quad$ If $\quad \mathfrak{M}(L \otimes M)=\mathfrak{M}(L) \otimes \mathfrak{M}(M) \quad(=\{x \otimes y \mid x \in \mathfrak{M}(L)$, $y \in \mathfrak{M}(M)\})$ for any positive lattice $M$, then $L$ is called of $E$-type. $\tilde{L}$ is a submodule of $L$ spanned by $\mathfrak{M}(L)$. If $L \cong L_{1} \otimes L_{2}$ implies $\operatorname{rank} L_{1}$ or $\operatorname{rank} L_{2}=1$, then we say that $L$ is indecomposable with respect to tensor product. $O(L)$ denotes the orthogonal group of $L$. If a positive lattice $X$ is isometric to $L \otimes K$ for a positive lattice $K$, then $X$ is, by definition, divided by $L$. These notations and terminology will be used through this paper.

In $\S 1$ we prove a theorem about weighted graphs. In $\S 2$ we improve Received August 13, 1979. 
a result in [4] and in $\S 3$ the above theorem is proved. In $\S 4$ examples of a lattice $L$ as in the theorem are given.

$\S 1$.

In this section we define a weighted graph and prove a fundamental theorem in this paper.

Definition. Let $A$ be a finite set and [ , ] be a mapping from $A \times A$ into $\{t \mid 0 \leq t \leq 1\}$ such that

(i) $\left[a, a^{\prime}\right]=1$ if and only if $a=a^{\prime}$, and

(ii) $\left[a, a^{\prime}\right]=\left[a^{\prime}, a\right]$ for $a, a^{\prime} \in A$.

Then we call $(A,[]$,$) or simply A$ a weighted graph.

Let $A$ be a weighted graph. $A$ is called connected unless there exist subsets $A_{1}, A_{2}$ of $A$ such that $A=A_{1} \cup A_{2}, A_{1} \cap A_{2}=\phi$ and $\left[a_{1}, a_{2}\right]=0$ for any $a_{i} \in A_{i}(i=1,2)$. If $A=\cup A_{i}$ (disjoint) satisfies

(i) $A_{i}$ is connected, and

(ii) $[a, b]=0$ if $a \in A_{i}, b \in A_{j}$ and $i \neq j$,

then each $A_{i}$ is called a connected component of $A$. Let $A, B$ be weighted graphs. For $(a, b),\left(a^{\prime}, b^{\prime}\right) \in A \times B$ we define $\left[(a, b),\left(a^{\prime}, b^{\prime}\right)\right]$ by $\left[a, a^{\prime}\right] \cdot\left[b, b^{\prime}\right]$. Then $A \times B$ becomes a weighted graph. If there exists a bijection $\sigma$ from $A$ on $B$ such that $\left[a, a^{\prime}\right]=\left[\sigma(a), \sigma\left(a^{\prime}\right)\right]\left(a, a^{\prime} \in A\right)$, then we say that $A, B$ are isometric and write $\sigma: A \cong B$.

LEMMA 1. Let $A, B, C$ be weighted graphs and assume that $A=\left\{e_{i}\right\}_{i=1}^{n}$ and $\sigma: A \times B \cong A \times C$. Take any element $b \in B$ and fix it. Define $f_{i} \in A$, $c_{i} \in C, g_{i j} \in A, b_{i j} \in B$ by

$$
\sigma\left(e_{i}, b\right)=\left(f_{i}, c_{i}\right) \text { and } \sigma\left(g_{i j}, b_{i j}\right)=\left(f_{i}, c_{j}\right) .
$$

Then we have $\left[e_{i}, e_{j}\right]=0$ if $b_{i j} \neq b$.

Proof. Set $a_{i j}=\left[e_{i}, e_{j}\right]$. Then $a_{i j}=a_{j i}$ and

$$
a_{i j}=\left[f_{i}, f_{j}\right]\left[c_{i}, c_{j}\right] \text {. }
$$

Fix any integer $k(1 \leq k \leq n)$ and define $e_{s}^{\prime} \in A, b_{s} \in B$ by $\sigma\left(e_{s}^{\prime}, b_{s}\right)=\left(f_{k}, c_{s}\right)$ $(1 \leq s \leq n)$. Put $S=\left\{s \mid b_{s} \neq b\right\}$. If $S \neq \phi$, then we take integers $u, m$ such that

$$
a_{u m}=\max _{\substack{i: f_{i} f_{k} \\ s \in S}} a_{i s} \text { and } f_{u}=f_{k}, \quad m \in S .
$$

If $a_{u m}=0$ can be shown, then the lemma will be proved. Assume $a_{u m} \neq 0$ and put $b_{m}=b^{\prime}, e_{m}^{\prime}=e_{p}$. Then we have 


$$
\sigma\left(e_{p}, b^{\prime}\right)=\left(f_{k}, c_{m}\right), \quad b^{\prime} \neq b .
$$

Since $\sigma\left(e_{i}, b\right)=\left(f_{i}, c_{i}\right)$, we have

$$
\begin{aligned}
a_{i p}\left[b, b^{\prime}\right] & =\left[f_{i}, f_{k}\right]\left[c_{i}, c_{m}\right], \\
a_{i p}\left[b, b^{\prime}\right] & =\left[c_{i}, c_{m}\right] \quad \text { if } f_{i}=f_{k}, \\
a_{m p}\left[b, b^{\prime}\right] & =\left[f_{m}, f_{k}\right] .
\end{aligned}
$$

Hence $f_{u}=f_{k}$ implies

$$
\begin{array}{rlrl}
a_{u m} & =\left[f_{u}, f_{m}\right]\left[c_{u}, c_{m}\right] & & \text { by (0) } \\
& =\left[f_{k}, f_{m}\right]\left[c_{u}, c_{m}\right] & \\
& =a_{m p} a_{u p}\left[b, b^{\prime}\right]^{2} & \text { by (2), (3). }
\end{array}
$$

$$
a_{u m}=a_{m p} a_{u p}\left[b, b^{\prime}\right]^{2} \text {. }
$$

Suppose $f_{p}=f_{k}$. Then $\sigma\left(e_{p}, b^{\prime}\right)=\left(f_{k}, c_{m}\right)=\left(f_{p}, c_{m}\right)$ implies $a_{u m} \geq a_{p m}$. Hence we have

$$
\begin{aligned}
0<a_{u m} & =a_{m p} a_{u p}\left[b, b^{\prime}\right]^{2} \quad \text { by (4) } \\
& \leq a_{u m} a_{u p}\left[b, b^{\prime}\right]^{2} \\
& \leq a_{u m}
\end{aligned}
$$

This yields $a_{u p}\left[b, b^{\prime}\right]^{2}=1$ and $\left[b, b^{\prime}\right]=1$. This contradicts $b \neq b^{\prime}$. Therefore we get $f_{p} \neq f_{k}$. Suppose $p \in S$; then $a_{u p} \leq a_{u m}$ holds by definition. Hence we have

$$
\begin{aligned}
0<a_{u m} & =a_{m p} a_{u p}\left[b, b^{\prime}\right]^{2} \quad \text { by }(4) \\
& \leq a_{m p} a_{u m}\left[b, b^{\prime}\right]^{2} \\
& \leq a_{u m} .
\end{aligned}
$$

This implies $\left[b, b^{\prime}\right]=1$ and it contradicts $b \neq b^{\prime}$. Hence we get $p \notin S$ and by definition of $S$ there exists an integer $t$ such that $\sigma\left(e_{t}, b\right)=\left(f_{k}, c_{p}\right)$. On the other hand $\sigma\left(e_{t}, b\right)=\left(f_{t}, c_{t}\right)$ holds. Hence we get $f_{k}=f_{t}, c_{p}=c_{t}$ and by (2)

$$
a_{t p}\left[b, b^{\prime}\right]=\left[c_{t}, c_{m}\right]
$$

and by (1)

$$
\left[b, b^{\prime}\right]=\left[f_{p}, f_{k}\right]\left[c_{p}, c_{m}\right]
$$

From these follows 


$$
\begin{aligned}
{\left[c_{p}, c_{m}\right] } & =\left[c_{t}, c_{m}\right] \\
& =a_{t p}\left[b, b^{\prime}\right] \\
& =a_{t p}\left[f_{p}, f_{k}\right]\left[c_{p}, c_{m}\right] .
\end{aligned}
$$

If $\left[c_{p}, c_{m}\right] \neq 0$, then $a_{t p}\left[f_{p}, f_{k}\right]=1$ and this contradicts $f_{p} \neq f_{k}$. Hence we have $\left[c_{p}, c_{m}\right]=0$ and $\left[b, b^{\prime}\right]=0$ by (5) and $\left[f_{m}, f_{k}\right]=0$ by (3), and $a_{u m}=$ $\left[f_{u}, f_{m}\right]\left[c_{u}, c_{m}\right]=\left[f_{k}, f_{m}\right]\left[c_{u}, c_{m}\right]=0$. This contradicts our assumption $a_{u m} \neq$ 0 . Thus we have proved $a_{u m}=0$.

Q.E.D.

Theorem 1. Let $A, B, C$ be weighted graphs and assume that $A=$ $\left\{e_{i}\right\}_{i=1}^{n}$ is connected and $\sigma: A \times B \cong A \times C$. Take any element $b \in B$ and put $\sigma\left(e_{i}, b\right)=\left(f_{i}, c_{i}\right)$. Then we have

$$
A \cong\left\{\sigma\left(e_{i}, b\right) \mid 1 \leq i \leq n\right\}=\left\{f_{i} \mid 1 \leq i \leq n\right\} \times\left\{c_{i} \mid 1 \leq i \leq n\right\}
$$

Proof. Put $C_{i}=\left\{c_{k} \mid k\right.$ satisfies $\left.f_{k}=f_{i}\right\}$ for $1 \leq i \leq n$, and denote by $\tilde{C}_{i}$ a connected component of $C_{i}$ which contains $c_{i}$. Suppose $\left[e_{i}, e_{j}\right]=$ $\left[f_{i}, f_{j}\right]\left[c_{i}, c_{j}\right] \neq 0$. We will show $\tilde{C}_{i}=\tilde{C}_{j}$. Since $\left[e_{i}, e_{j}\right] \neq 0$, Lemma 1 implies that there exists an element $e_{t} \in A$ such that $\sigma\left(e_{t}, b\right)=\left(f_{i}, c_{j}\right)$. Hence we have $f_{t}=f_{i}, c_{t}=c_{j}$ since $\sigma\left(e_{t}, b\right)=\left(f_{t}, c_{t}\right)$. By definition of $C_{i}$ we have $c_{j}=c_{t} \in C_{i}$ and hence $c_{j} \in \tilde{C}_{i}$ since $c_{i} \in \tilde{C}_{i}$ and $\left[c_{j}, c_{i}\right] \neq 0$. Thus we have proved $\tilde{C}_{i} \cap \tilde{C}_{j} \neq \phi$. Take any element $x \in \tilde{C}_{i} \cap \tilde{C}_{j}$; then there exists $u$ such that $x=c_{u}$ and $f_{u}=f_{i}$ since $x \in C_{i}$. Take any $y \in \tilde{C}_{j}$ such that $[y, x]$ $\neq 0$. Then $y$ can be written $y=c_{k}$ with $f_{k}=f_{j} . \quad\left[e_{u}, e_{k}\right]=\left[f_{u}, f_{k}\right]\left[c_{u}, c_{k}\right]=$ $\left[f_{i}, f_{j}\right][x, y] \neq 0$ yields that $\sigma\left(e_{s}, b\right)=\left(f_{u}, c_{k}\right)$ for some $s$. From $f_{s}=f_{u}=f_{i}$, $\left[c_{s}, c_{u}\right]=\left[c_{k}, c_{u}\right] \neq 0$ follows that $y=c_{k}=c_{s} \in \tilde{C}_{i}$ since $c_{u}=x \in \tilde{C}_{i}, c_{s} \in C_{i}$ and $\tilde{C}_{i}$ is a connected component of $C_{i}$. Thus we have shown that if $[x, y] \neq 0$ for $x \in \tilde{C}_{i} \cap \tilde{C}_{j}, y \in \tilde{C}_{j}$, then $y \in \tilde{C}_{i}$ holds. This implies $\tilde{C}_{j} \subset \tilde{C}_{i}$ and similarly $\tilde{C}_{i} \subset \tilde{C}_{j}$ and hence $\tilde{C}_{i}=\tilde{C}_{j}$ if $\left[e_{i}, e_{j}\right] \neq 0$. Since $A$ is connected we get $\tilde{C}_{1}=\cdots=\tilde{C}_{n}$. Take any $s, t(1 \leq s, t \leq n)$. From $c_{t} \in \tilde{C}_{t}$ $=\tilde{C}_{s} \subset C_{s}$ follows that there exists $i$ such that $c_{t}=c_{i}$ and $f_{i}=f_{s}$. Hence $\left(f_{s}, c_{t}\right)=\left(f_{i}, c_{i}\right)=\sigma\left(e_{i}, b\right)$ holds.

Q.E.D.

\section{$\S 2$.}

Let $L$ be an indecomposable positive lattice which satisfies the following condition $\left(\mathrm{A}^{\prime}\right)$.

$\left(\mathrm{A}^{\prime}\right)$ For any given positive lattices $M, N$ and for any isometry $\sigma$ from $L \otimes M$ on $L \otimes N$ which satisfies that $\sigma(L \otimes m)=L \otimes n(m \in M, n \in N)$ implies $m=0, n=0$, there exists a finite subset $\left\{v_{1}, \cdots, v_{m}\right\}$ of $L$ (depending on $M, N, \sigma)$ such that 
(1) each $v_{i}$ is primitive in $L$ and a submodule spanned by $\left\{v_{1}, \cdots, v_{m}\right\}$ of $L$ is of finite index in $L$,

(2) putting $M_{v_{i}}=\left\{m \in M \mid \sigma(L \otimes m) \subset v_{i} \otimes N\right\}$,

$$
N_{v_{i}}=\left\{n \in N \mid \sigma^{-1}(L \otimes n) \subset v_{i} \otimes M\right\},
$$

we have $\operatorname{rank} M_{v_{i}}=\operatorname{rank} N_{v_{i}}=\operatorname{rank} M / \operatorname{rank} L$, and

(3) $\sigma\left(\boldsymbol{Q}\left(v_{i} \otimes M_{v_{i}}\right)\right)=\boldsymbol{Q}\left(v_{i} \otimes N_{v_{i}}\right)$.

Through this section the above $L$ is fixed.

Lemma 2. Let $M, N, \sigma, v_{i}, M_{v_{i}}, N_{v_{i}}$ be those as in the condition ( $\left.\mathrm{A}^{\prime}\right)$. Then $M, N$ are isometric and they are divided by $L$, and $\sigma\left(L \otimes M_{v_{i}}\right)=v_{i} \otimes N$.

Proof. By definition $M_{v_{i}}, N_{v_{i}}$ are direct summands (as modules) of $M, N$ respectively, and $\sigma\left(L \otimes M_{v_{i}}\right) \subset v_{i} \otimes N, \quad \sigma^{-1}\left(L \otimes N_{v_{i}}\right) \subset v_{i} \otimes M$ and rank $\sigma\left(L \otimes M_{v_{i}}\right)=\operatorname{rank}\left(v_{i} \otimes N\right)$, rank $\sigma^{-1}\left(L \otimes N_{v_{i}}\right)=\operatorname{rank}\left(v_{i} \otimes M\right)$ imply $\sigma\left(L \otimes M_{v_{i}}\right)=v_{i} \otimes N$ and $\sigma^{-1}\left(L \otimes N_{v_{i}}\right)=v_{i} \otimes M$ since they are direct summands in $L \otimes N, L \otimes M$ respectively. This implies that $M, N$ are divided by $L$. From (3) follows $\sigma\left(v_{i} \otimes M_{v_{i}}\right)=v_{i} \otimes N_{v_{i}}$ since they are direct summands of $L \otimes N$. Hence we can define an isometry $\mu_{i}: M_{v_{i}} \cong N_{v_{i}}$ by $\sigma\left(v_{i} \otimes m\right)=v_{i} \otimes \mu_{i}(m)$ for $m \in M_{v_{i}}$. For $m_{i} \in M_{i}, m_{j} \in M_{j}$ we show $B\left(m_{i}, m_{j}\right)$ $=B\left(\mu_{i}\left(m_{i}\right), \mu_{j}\left(m_{j}\right)\right)$ where $B$ stands for bilinear forms associated with quadratic modules.

$$
\begin{aligned}
B\left(v_{i}, v_{j}\right) B\left(m_{i}, m_{j}\right) & =B\left(v_{i} \otimes m_{i}, v_{j} \otimes m_{j}\right) \\
& =B\left(\sigma\left(v_{i} \otimes m_{i}\right), \sigma\left(v_{j} \otimes m_{j}\right)\right) \\
& =B\left(v_{i} \otimes \mu_{i}\left(m_{i}\right), v_{j} \otimes \mu_{j}\left(m_{j}\right)\right) \\
& =B\left(v_{i}, v_{j}\right) B\left(\mu_{i}\left(m_{i}\right), \mu_{j}\left(m_{j}\right)\right) .
\end{aligned}
$$

Hence we have $B\left(m_{i}, m_{j}\right)=B\left(\mu_{i}\left(m_{i}\right), \mu_{j}\left(m_{j}\right)\right)$ if $B\left(v_{i}, v_{j}\right) \neq 0$. Suppose $B\left(v_{i}, v_{j}\right)=0$, then we have

$$
\begin{aligned}
B\left(L \otimes M_{v_{i}}, L \otimes M_{v_{j}}\right) & =B\left(\sigma\left(L \otimes M_{v_{i}}\right), \sigma\left(L \otimes M_{v_{j}}\right)\right) \\
& =B\left(v_{i} \otimes N, v_{j} \otimes N\right) \\
& =0 \\
B\left(L \otimes \mu_{i}\left(M_{v_{i}}\right), L \otimes \mu_{j}\left(M_{v_{j}}\right)\right) & =B\left(L \otimes N_{v_{i}}, L \otimes N_{v_{j}}\right) \\
& =B\left(\sigma^{-1}\left(L \otimes N_{v_{i}}\right), \sigma^{-1}\left(L \otimes N_{v_{j}}\right)\right) \\
& =B\left(v_{i} \otimes M, v_{j} \otimes M\right) \\
& =0 .
\end{aligned}
$$

Hence $B\left(M_{v_{i}}, M_{v_{j}}\right)=B\left(\mu_{i}\left(M_{v_{i}}\right), \mu_{j}\left(M_{v_{j}}\right)\right)=0$ follows. Thus we have proved $B\left(m_{i}, m_{j}\right)=B\left(\mu_{i}\left(m_{i}\right), \mu_{j}\left(m_{j}\right)\right)$ for $m_{i} \in M_{v_{i}}, m_{j} \in M_{v_{j}}$. By (1) we can choose 
a subset of $\left\{v_{1}, \cdots, v_{m}\right\}$, say $\left\{v_{1}, \cdots, v_{n}\right\}$, so that it is a basis of $\boldsymbol{Q} L$. Then $\sigma\left(L \otimes M_{v_{i}}\right)=v_{i} \otimes N$ implies that $\sum_{i=1}^{n} M_{v_{i}}$ is a direct sum and $\left[M: \sum_{i=1}^{n} M_{v_{i}}\right]$ $<\infty$. Hence a linear mapping $\mu$ from $\boldsymbol{Q} M$ to $\boldsymbol{Q} N$ defined by $\mu\left(\sum_{i=1}^{n} m_{i}\right)$ $=\sum_{i=1}^{n} \mu_{i}\left(m_{i}\right)\left(m_{i} \in M_{v_{i}}\right)$ becomes an isometry from $\boldsymbol{Q} M$ on $\boldsymbol{Q} N$. We have only to show $\mu(M)=N$. Take a basis $\left\{e_{i}\right\}$ of $L$ and put

$$
\boldsymbol{e}_{i}=\sum_{j=1}^{n} a_{i j} v_{j}, \quad v_{i}=\sum_{j=1}^{n} b_{i j} e_{j} \quad\left(a_{i j}, b_{i j} \in \mathbf{Q}\right) .
$$

$\sum_{k=1}^{n} b_{i k} a_{k j}=\delta_{i j}$ (Kronecker's delta) is obvious. Take any element $m=$ $\sum_{i=1}^{n} m_{i}\left(m_{i} \in \boldsymbol{Q} M_{v_{i}}\right)$ of $M$ and put $\sigma\left(v_{j} \otimes m_{i}\right)=v_{i} \otimes n_{j i}\left(n_{j i} \in \boldsymbol{Q} N\right)$; then $n_{i i}=\mu\left(m_{i}\right)$ follows and

$$
\begin{aligned}
\sigma\left(e_{k} \otimes m\right) & =\sigma\left(\sum_{i, j} a_{k j} v_{j} \otimes m_{i}\right) \\
& =\sum_{i, j} a_{k j} v_{i} \otimes n_{j i} \\
& =\sum_{t} e_{t} \otimes\left(\sum_{i, j} a_{k j} b_{i t} n_{j i}\right) .
\end{aligned}
$$

Since $\sigma\left(e_{k} \otimes m\right) \in L \otimes N$, we get $\sum_{i, j} a_{k j} b_{i k} n_{j i} \in N$. Summing up with respect to $k$, we have

$$
\mu(m)=\sum \mu\left(m_{i}\right)=\sum n_{i i} \in N .
$$

Thus $\mu(M) \subset N$ is proved. Since discriminants of $M, N$ are equal, we have $\mu(M)=N$.

Q.E.D.

Lemma 3. Let $K, X, Y$ be positive lattices and assume that $K$ is indecomposable and $\sigma: K \otimes X \cong K \otimes Y$. Then there exist submodules $M_{0}, M$ of $X$ and $N_{0}, N$ of $Y$ such that

(i) $M_{0}, M, N_{0}, N$ are direct summands of $X, Y$ respectively and $\left[X: M_{0} \perp M\right],\left[Y: N_{0} \perp N\right]<\infty$, and

$$
\sigma\left(K \otimes M_{0}\right)=K \otimes N_{0}, \quad \sigma(K \otimes M)=K \otimes N,
$$

(ii) if $\sigma(K \otimes m)=K \otimes n(m \in M, n \in N)$, then $m=0$ and $n=0$, and

(iii) there exist orthogonal decompositions

$$
M_{0}=\stackrel{1}{i=1}_{i}^{t} M_{0, i}, \quad N_{0}=\stackrel{1}{i=1}_{0, i}
$$

such that $\sigma\left(K \otimes M_{0, i}\right)=K \otimes N_{0, i}(1 \leq i \leq t)$ and

$$
\left.\sigma\right|_{K \otimes M_{0, i}}=\alpha_{i} \otimes \beta_{i}
$$

where $\alpha_{i} \in O(K), \beta_{i}: M_{0, i} \cong N_{0, i}$. 
Proof. Suppose that $m_{1}, \cdots, m_{r}$ are linearly independent elements of $X$ so that there exist elements $n_{i} \in Y$ such that $\sigma\left(K \otimes m_{i}\right)=K \otimes n_{i}$. We may assume that $r$ is maximal. Then we put $M=Z\left[m_{1}, \cdots, m_{r}\right]^{\perp}, N=$ $Z\left[n_{1}, \cdots, n_{r}\right]^{\perp}$ and $M_{0}=M^{\perp}, N_{0}=N^{\perp}$. Clearly (i), (ii) are satisfied. (iii) follows from Lemma 1 in $\S 3$ in [2].

Q.E.D.

Lemma 4. $L \otimes L$ is indecomposable and $O(L \otimes L)$ is generated by $O(L)$ and an interchange of $L$ 's.

Proof. Take an isometry $\sigma$ of $L \otimes L$. Suppose that there exist $x, y$ $\in L, \quad x \neq 0$ such that $\sigma(L \otimes x)=L \otimes y$. Supposing $K=X=Y=L$ in Lemma 3 , a submodule of $L$ corresponding to $M$ of $X$ in Lemma 3 is $\{0\}$ since its $\operatorname{rank}(<\operatorname{rank} L)$ is divided by $\operatorname{rank} L$ by Lemma 2 . Hence we have $\sigma \in O(L) \otimes O(L)$ by Lemma 3. Suppose that there are no such elements $x, y$ in $L$. Then, by Lemma 2, there is an element $v \in L$ such that

$$
\sigma\left(L \otimes L_{v}\right)=v \otimes L,
$$

where $L_{v}=\{x \in L \mid \sigma(L \otimes x) \subset v \otimes L\}$. Since rank $L_{v}=1$, there is an element $u$ such that $L_{v}=Z[u]$. Then $\mu \sigma(L \otimes u)=L \otimes v$ holds where $\mu \epsilon$ $O(L \otimes L)$ is defined by $\mu(x \otimes y)=y \otimes x(x, y \in L)$. Hence $\mu \sigma \in O(L) \otimes O(L)$ follows as above. The indecomposability of $L \otimes L$ is proved quite similarly as in the proof of Lemma 4 in [4].

Q.E.D.

LEMMA 5. $\otimes^{m} L$ is indecomposable provided that the orthogonal group $O\left(\otimes^{m} L\right)$ is generated by $O(L)$ and interchanges of $L$ 's and that $\otimes^{m-1} L$ is indecomposable.

Proof. The proof is identical with that of Lemma 5 in [4].

Theorem 2. Let $X$ be an indecomposable positive lattice. Then we have

(i) for any positive lattice $Y, L \otimes X \cong L \otimes Y$ implies $X \cong Y$,

(ii) if $X=\otimes^{t} L \otimes X^{\prime}$ where $X^{\prime}$ is a positive lattice which is not divided by $L$, then $O(L \otimes X)$ is generated by $O(L), O\left(X^{\prime}\right)$ and interchanges of $L$ 's,

(iii) $L \otimes X$ is indecomposable.

Proof. We induct on $\operatorname{rank} X$. In case of $\operatorname{rank} X=1$ our assertion is obvious. Suppose $\operatorname{rank} X=k+1$. Let $Y$ be a positive lattice and $\sigma: L \otimes X \cong L \otimes Y$. Let $M_{0}, M$ (resp. $N_{0}, N$ ) be submodules of $X$ (resp. $Y$ ) 
as in Lemma 3 for $K=L$. If $M=X$ (resp. $M_{0}=X$ ), then $X \cong Y$ follows from Lemma 2 (resp. Lemma 3). Hence we may assume $M_{0} \neq\{0\}, M \neq\{0\}$. Lemma 2 implies $M \cong N$. Hence we may assume $M=N=\perp K_{i}$ where $K_{i}$ is indecomposable and suppose $K_{i} \cong \otimes^{r_{i}} L \otimes K_{i}^{\prime}$ where $K_{i}^{\prime}$ is not divided by $L$. Since $\operatorname{rank} K_{i} \leq \operatorname{rank} M \leq k$, the inductive assumption implies that $L \otimes K_{i}$ is indecomposable and $O\left(L \otimes K_{i}\right)$ is generated by $O(L)$, $O\left(K_{i}^{\prime}\right)$ and interchanges of $L$ 's, identifying $K_{i}$ and $\otimes^{r_{i}} L \otimes K_{i}^{\prime}$. Hence, noting $L \otimes M \cong L \otimes N \cong \perp L \otimes K_{i}$, as in 2 in [4] for any basis $\left\{u_{1}, \cdots, u_{n}\right\}$ of $L$ we have

$$
\begin{aligned}
\sigma\left(L \otimes M_{u_{i}}\right) & =u_{i} \otimes N, \quad \sigma^{-1}\left(L \otimes N_{u_{i}}\right)=u_{i} \otimes M, \\
\sigma\left(u_{i} \otimes M_{u_{i}}\right) & =u_{i} \otimes N_{u_{i}},
\end{aligned}
$$

where

$$
\begin{aligned}
& M_{u_{i}}=\left\{m \in M \mid \sigma(L \otimes m) \subset u_{i} \otimes N\right\}, \\
& N_{u_{i}}=\left\{n \in N \mid \sigma^{-1}(L \otimes n) \subset u_{i} \otimes M\right\} .
\end{aligned}
$$

Now $X=M_{0} \perp M, Y=N_{0} \perp N$ are proved quite similarly as in the proof of Theorem in $\S 1$ in [3]. This is a contradiction since $X$ is indecomposable. Thus (i) is proved. Let $X$ be a positive lattice as in (ii). Assume that there exists an isometry $\sigma \in O(L \otimes X)$ which is not contained in a subgroup of $O(L \otimes X)$ generated by $O(L), O\left(X^{\prime}\right)$ and interchanges of $L$ 's. Suppose that there exist $x, y \in X$ such that $\sigma(L \otimes x)=L \otimes y$. We define $M_{0}, M, N_{0}, N$ as in Lemma 3 for $K=L, Y=X$. Then $X=M_{0} \perp M$ holds as above. Since $M_{0} \neq\{0\}$ and $X$ is indecomposable, we have $X=M_{0}$ and Lemma 3 implies $\sigma \in O(L) \otimes O(X)$. If $X$ is divided by $L$, that is, $t \geq 1$, then $O(X)=O\left(L \otimes\left(\otimes^{t-1} L \otimes X^{\prime}\right)\right)$ is generated by $O(L)$ and $O\left(X^{\prime}\right)$ and interchanges of $L$ 's since $\otimes^{t-1} L \otimes X^{\prime}$ is indecomposable and rank $\otimes^{t-1} L$ $\otimes X^{\prime} \leq k$. Thus $\sigma$ is contained in a subgroup generated by $O(L), O\left(X^{\prime}\right)$ and interchanges of $L$ 's in $O(L \otimes X)$. This is a contradiction. Therefore there exist no such elements $x, y$. Hence from Lemma 2 follows that $t \geq 1$ and there exists non-zero $v \in L$ such that $\sigma\left(L \otimes X_{v}\right)=v \otimes X$ where $X_{v}=$ $\{x \in X \mid \sigma(L \otimes x) \subset v \otimes X\}$ by the assumption on $L$. Define $\mu_{2} \in O(L \otimes X)$ by $\mu_{2}(x \otimes y \otimes z)=y \otimes x \otimes z\left(x, y \in L, z \in \otimes^{t-1} L \otimes X^{\prime}\right)$; then $\mu_{2} \sigma\left(L \otimes X_{v}\right)=$ $L \otimes v \otimes \otimes{ }^{t-1} L \otimes X^{\prime}$. If there exist $x \in X_{v}, y \in v \otimes \otimes \otimes^{t-1} L \otimes X^{\prime}$ such that $x \neq 0$ and $\mu_{2} \sigma(L \otimes x)=L \otimes y$, then $\mu_{2} \sigma \in O(L \otimes X)$ must be contained in a subgroup generated by $O(L), O\left(X^{\prime}\right)$ and interchanges of $L$ 's as above. This is also a contradiction. Repeating this operation we get, as in 1.6 in [4], 


$$
\mu_{t+1} \cdots \mu_{2} \sigma\left(L \otimes X_{\left.v, \cdots, v^{\prime \cdots .}\right)}=L \otimes v \otimes \cdots \otimes v^{\prime \cdots \prime} \otimes X^{\prime},\right.
$$

where $\mu_{j} \in O(L \otimes X)$ is defined by

$$
\begin{aligned}
& \mu_{j}\left(x_{1} \otimes \cdots \otimes x_{j} \otimes \cdots \otimes x_{t+1} \otimes y\right) \\
& \quad=x_{j} \otimes \cdots \otimes x_{1} \otimes \cdots \otimes x_{t+1} \otimes y \quad\left(x_{i} \in L, y \in X^{\prime}\right) .
\end{aligned}
$$

If there exist $x \in X_{v}, \cdots, v^{\prime} \cdots, y \in v \otimes \cdots \otimes X^{\prime}$ such that $x \neq 0$ and $\mu_{t+1} \cdots$ $\mu_{2} \sigma(L \otimes x)=L \otimes y$, then $\mu_{t+1} \cdots \mu_{2} \sigma$ is contained in a subgroup generated by $O(L), O\left(X^{\prime}\right)$ and interchanges of $L$ 's. This is a contradiction. Hence Lemma 2 yields that $v \otimes \cdots \otimes v^{\prime \cdots \prime} \otimes X^{\prime}$ is divided by $L$. This contradicts the assumption on $X^{\prime}$. Thus the proof of (ii) is completed. Let $X$ be a positive lattice as in (ii). Then $O(L \otimes X)=O\left(\otimes^{t+1} L\right) \otimes O\left(X^{\prime}\right)$ has been proved as above. To complete the proof of (iii) we have only to show that $\otimes^{t+1} L$ is indecomposable by virtue of Lemma 3 in [4]. Since $X$ is indecomposable, $\otimes^{t} L$ is also indecomposable. By virtue of (ii) $O\left(\otimes^{t+1} L\right)$ is generated by $O(L)$ and interchanges by $L$ 's. Hence Lemma 5 implies that $\otimes^{t+1} L$ is indecomposable.

Q.E.D.

Remark. By (i), (iii) and Theorem in 105:1 in [5] $L \otimes X \cong L \otimes Y$ implies $X \cong Y$ for any (not necessarily indecomposable) positive lattices $X, Y$.

$\S 3$.

Through this section we fix any positive lattice $L$ of E-type such that $[L: \tilde{L}]<\infty$ and $\tilde{L}$ is indecomposable.

Lemma 6. Let $M, N$ be positive lattices and assume $\sigma: L \otimes M \cong L \otimes N$. Then for each $m \in \mathfrak{M}(M)$ we have $\sigma(L \otimes m)=F \otimes G$, where $F, G$ are submodules of $L, N$ respectively and $m(F)=m(L)$, and $m(G)=m(N)$.

Proof. Let $X$ be a positive lattice. For $x, y \in \mathfrak{M}(X) / \pm$, we put $[x, y]$ $=|B(x, y)| \mid m(X)$. Then $\mathfrak{M}(X) \mid \pm$ becomes a weighted graph and $\tilde{X}$ is indecomposable if and only if $\mathfrak{M}(X) / \pm$ is connected. Put $A=\mathfrak{M}(L) / \pm$, $B=\mathfrak{M}(M) / \pm, C=\mathfrak{M}(N) / \pm$. Since $L$ is of $E$-type, we have $\mathfrak{M}(L \otimes M)=$ $\mathfrak{M}(L) \otimes \mathfrak{M}(M), \mathfrak{M}(L \otimes N)=\mathfrak{M}(L) \otimes \mathfrak{M}(N)$ and $\sigma$ induces an isometry from $A \times B$ on $\mathrm{A} \times C$. By Theorem 1 there exist subsets $F^{\prime} \subset A, G^{\prime} \subset C$ such that $\sigma(A, m)=\left(F^{\prime}, G^{\prime}\right)$. Denoting by $F_{0}, G_{0}$ submodules of $L, N$ spanned by $F^{\prime} \subset \mathfrak{M}(L) / \pm, G^{\prime} \subset \mathfrak{M}(N) / \pm$ respectively, we have $\sigma(\tilde{L} \otimes m)=F_{0} \otimes G_{0}$ and $m\left(F_{0}\right)=m(L), m\left(G_{0}\right)=m(N)$. Put $F=\boldsymbol{Q} F_{0} \cap L, G=\boldsymbol{Q} G_{0} \cap N$; then 
$\left[F: F_{0}\right],\left[G: G_{0}\right]<\infty, m(F)=m(L), m(G)=m(N)$ and $\sigma(L \otimes m), F \otimes G$ are direct summands of $L \otimes N$. Hence $\sigma(L \otimes m)=F \otimes G$ follows. $\quad$ Q.E.D.

THEOREM 3. If $L \cong L_{1} \otimes L_{2}$ for positive lattices $L_{1}, L_{2}$, then $L_{1}, L_{2}$ are of E-type, $\left[L_{1}: \tilde{L}_{1}\right],\left[L_{2}: \tilde{L}_{2}\right]<\infty$ and $\tilde{L}_{1}, \tilde{L}_{2}$ are indecomposable.

Proof. Define $\sigma \in O\left(L_{1} \otimes L_{2} \otimes L_{2}\right)$ by $\sigma(x \otimes y \otimes z)=x \otimes z \otimes y\left(x \in L_{1}\right.$, $\left.y, z \in L_{2}\right)$. For each $m \in \mathfrak{M}\left(L_{2}\right) \sigma\left(\left(L_{1} \otimes L_{2}\right) \otimes m\right)=\left(L_{1} \otimes m\right) \otimes L_{2}$ holds. Applying Lemma 6 in case of $M=N=L_{2}$, we have $m\left(L_{1} \otimes m\right)=m(L)$. From Proposition 2 in [1] follows that $L_{1} \otimes m$ is of $E$-type. Hence $L_{1}$ is of $E$-type. Similarly $L_{2}$ is of $E$-type. $\mathfrak{M}(L)=\mathfrak{M}\left(L_{1}\right) \otimes \mathfrak{M}\left(L_{2}\right)$ implies $\left[L_{1}: \tilde{L}_{1}\right],\left[L_{2}: \tilde{L}_{2}\right]<\infty$ and $\tilde{L}_{1}, \tilde{L}_{2}$ are indecomposable since $[L: \tilde{L}]<\infty$ and $\tilde{L}$ is indecomposable.

Q.E.D.

THEOREM 4. Assume that $L$ is indecomposable with respect to tensor product. Then $L$ satisfies the condition $\left(\mathrm{A}^{\prime}\right)$ in $\S 2$.

Proof. Suppose that $L$ is decomposable and $L=L_{1} \perp L_{2}\left(L_{1}, L_{2} \neq 0\right)$. Each $x \in \mathfrak{M}(L)$ is contained in $L_{1}$ or $L_{2}$. If $\mathfrak{M}(L) \cap L_{1}=\phi$, then $\mathfrak{M}(L) \subset$ $L_{2}$ and hence $\tilde{L} \subset L_{2}$ and $\operatorname{rank} L \leq \operatorname{rank} L_{2}$. This is a contradiction. Hence we have $\mathfrak{M}(L) \cap L_{i} \neq \phi(i=1,2)$ and then $\tilde{L}$ spanned by $\mathfrak{M}(L)$ is decomposable. This contradicts our assumption on $L$. Thus $L$ is indecomposable. Set $\mathfrak{M}(L)=\left\{ \pm v_{1}, \cdots, \pm v_{m}\right\}$. We show that the condition $\left(\mathrm{A}^{\prime}\right)$ is satisfied for the subset $\left\{v_{1}, \cdots, v_{m}\right\}$ of $L$ by induction with respect to rank $M$. The first condition of $\left(\mathrm{A}^{\prime}\right)$ follows from our assumption on $L$. Let $M, N$ be positive lattices and suppose that for $\sigma: L \otimes M \cong L \otimes N$, $\sigma(L \otimes m)=L \otimes n(m \in M, n \in N)$ implies $m=0, n=0$. Since $L$ is of $E$ type, we have $\sigma(\mathfrak{M}(L) \otimes \mathfrak{M}(M))=\mathfrak{M}(L) \otimes \mathfrak{M}(N)$ and hence $\sigma(\tilde{L} \otimes \tilde{M})=\tilde{L}$ $\otimes \tilde{N}$. Put $\tilde{M}^{\perp}=M^{\prime}, \tilde{N}^{\perp}=N^{\prime}, M^{\prime \prime}=M^{\perp \perp}(\neq\{0\}), N^{\prime \prime}=N^{\prime \perp}(\neq\{0\})$; then we have $\left[M: M^{\prime} \perp M^{\prime \prime}\right],\left[N: N^{\prime} \perp N^{\prime \prime}\right]<\infty, \sigma\left(L \otimes M^{\prime}\right)=L \otimes N^{\prime}$ and $\sigma(L$ $\left.\otimes M^{\prime \prime}\right)=L \otimes N^{\prime \prime}$ by virtue of $[L: \tilde{L}]<\infty$. Assume $M^{\prime} \neq 0$; then the inductive assumption implies $\operatorname{rank} M_{v_{i}}^{\prime}=\operatorname{rank} N_{v_{i}}^{\prime}=\operatorname{rank} M^{\prime} / \operatorname{rank} L$ and $\operatorname{rank} M_{v_{i}}^{\prime \prime}=\operatorname{rank} N_{v_{i}}^{\prime \prime}=\operatorname{rank} M^{\prime \prime} / \operatorname{rank} L$, where

$$
\begin{gathered}
M_{v_{i}}^{\prime}=\left\{m \in M^{\prime} \mid \sigma(L \otimes m) \subset v_{i} \otimes N^{\prime}\right\}, \\
N_{v_{i}}^{\prime}=\left\{n \in N^{\prime} \mid \sigma^{-1}(L \otimes n) \subset v_{i} \otimes M^{\prime}\right\}, \quad \text { and }
\end{gathered}
$$

$M_{v_{i}}^{\prime \prime}, N_{v_{i}}^{\prime \prime}$ are defined similarly for $M^{\prime \prime}, N^{\prime \prime}$. Moreover $M_{v_{i}}, N_{v_{i}}$ are defined similarly for $M, N$; then $M_{v_{i}} \supset M_{v_{i}}^{\prime} \perp M_{v_{i}}^{\prime \prime}$ and $N_{v_{i}} \supset N_{v_{i}}^{\prime} \perp N_{v_{i}}^{\prime \prime}$ are obvious. Hence $\operatorname{rank} M_{v_{i}} \geq \operatorname{rank} M / \operatorname{rank} L$ holds. Take any $i(1 \leq i \leq m)$ and a subset $S$ of $\left\{v_{1}, \cdots, v_{m}\right\}$ such that $S$ contains $v_{i}$ and $S$ is a basis of $\boldsymbol{Q} L$. 
We may assume $i=1, S=\left\{v_{1}, \cdots, v_{n}\right\}(n=\operatorname{rank} L)$. Then $\sigma\left(L \otimes M_{v_{i}}\right) \subset$ $v_{i} \otimes N$ and $\sigma\left(L \otimes \sum_{i=1}^{n} M_{v_{i}}\right) \subset \sum_{i=1}^{n} v_{i} \otimes N$ imply that $\sum_{i=1}^{n} M_{v_{i}}$ is a direct sum. Thus we have $\operatorname{rank} M \geq \sum_{i=1}^{n} \operatorname{rank} M_{v_{i}} \geq \sum_{i=1}^{n} \operatorname{rank} M / \operatorname{rank} L=$ $\operatorname{rank} M$ and hence $\operatorname{rank} M_{v_{1}}=\operatorname{rank} M / \operatorname{rank} L$. Hence $\operatorname{rank} M_{v_{i}}=\operatorname{rank} M /$ $\operatorname{rank} L$ for each $i$ and similarly rank $N_{v_{i}}=\operatorname{rank} N / \operatorname{rank} L$ hold. From this follows that $\boldsymbol{Q} M_{v_{i}}=\boldsymbol{Q} \boldsymbol{M}_{v_{i}}^{\prime} \perp \boldsymbol{Q} M_{v_{i}}^{\prime \prime}$ and $\boldsymbol{Q} N_{v_{i}}=\boldsymbol{Q} N_{v_{i}}^{\prime} \perp \boldsymbol{Q} N_{v_{i}}^{\prime \prime}$ and hence $\sigma\left(\boldsymbol{Q}\left(v_{i} \otimes M_{v_{i}}\right)\right)=\sigma\left(\boldsymbol{Q}\left(v_{i} \otimes M_{v_{i}}^{\prime}\right) \perp \boldsymbol{Q}\left(v_{i} \otimes M_{v_{i}}^{\prime \prime}\right)\right)=\boldsymbol{Q}\left(v_{i} \otimes N_{v_{i}}^{\prime}\right) \perp \boldsymbol{Q}\left(v_{i} \otimes N_{v_{i}}^{\prime \prime}\right)=$ $\boldsymbol{Q}\left(v_{i} \otimes N_{v_{i}}\right)$. Thus the condition (2), (3) are shown if $M^{\prime} \neq 0$. Suppose $M^{\prime}=0$; then $[M: \tilde{M}],[N: \tilde{N}]<\infty$ hold. For each $m \in \mathfrak{M}(M)$ Lemma 6 implies $\sigma(L \otimes m)=F \otimes G$ where $F, G$ are submodules of $L, N$ respectively and $m(F)=m(L)$. By the assumption on $L$ we get $\operatorname{rank} F$ or $\operatorname{rank} G=1$. rank $G=1$ implies $\sigma(L \otimes m)=L \otimes n$ for some $n \in N$ and it contradicts our assumption on $\sigma$. Hence we have $F=Z[v]$ for $v \in \mathfrak{M}(L)$.

Thus for each $m \in \mathfrak{M}(M)$ there exists $v \in \mathfrak{M}(L)$ such that $\sigma(L \otimes m) \subset v$ $\otimes N$

Take any $v_{i} \in \mathfrak{M}(L)$ and fix it. For $n \in \mathfrak{M}(N)$ suppose $\sigma(v \otimes m)=v_{i} \otimes n$ for $v \in \mathfrak{M}(L), m \in \mathfrak{M}(M)$. Since $\sigma(L \otimes m) \subset v_{j} \otimes N$ for $v_{j} \in \mathfrak{M}(L)$ as above, $v_{j}$ must be equal to $v_{i}$ and hence $\sigma(L \otimes m) \subset v_{i} \otimes N, m \in M_{v_{i}}$ and $m\left(M_{v_{i}}\right)$ $=m(M)$. Therefore $v_{i} \otimes n=\sigma(v \otimes m) \in \sigma\left(L \otimes M_{v_{i}}\right) \subset v_{i} \otimes N$ holds for each $n \in \mathfrak{M}(N)$. Thus we get $v_{i} \otimes \tilde{N} \subset \sigma\left(L \otimes M_{v_{i}}\right) \subset v_{i} \otimes N$. From $[N: \tilde{N}]<\infty$ follows $\operatorname{rank} M_{v_{i}}=\operatorname{rank} N / \operatorname{rank} L, m\left(M_{v_{i}}\right)=m(M)$ and $\left[M_{v_{i}}: \tilde{M}_{v_{i}}\right]<\infty$. Similarly rank $N_{v_{i}}=\operatorname{rank} M / \operatorname{rank} L$ follows.

For each $m \in \mathfrak{M}(M) \cap M_{v_{i}}=\mathfrak{M}\left(M_{v_{i}}\right)$ we put $\sigma\left(v_{i} \otimes m\right)=v_{i} \otimes n(n \in$ $\mathfrak{M}(N))$. Then we have $\sigma^{-1}(L \otimes n) \subset v_{i} \otimes M$ by $\sigma^{-1}\left(v_{i} \otimes n\right)=v_{i} \otimes m$. Hence $n \in N_{v_{i}}$ follows. Conversely $n \in \mathfrak{M}(N) \cap N_{v_{i}}=\mathfrak{M}\left(N_{v_{i}}\right)$ implies $\sigma^{-1}\left(v_{i} \otimes n\right)=$ $v_{i} \otimes m$ for $m \in \mathfrak{M}(M)$ and $\sigma(L \otimes m) \subset v_{i} \otimes N$ by $\sigma\left(v_{i} \otimes m\right)=v_{i} \otimes n$. Hence we have $m \in M_{v_{i}}$ and $\sigma\left(v_{i} \otimes \mathfrak{M}\left(M_{v_{i}}\right)\right)=v_{i} \otimes \mathfrak{M}\left(N_{v_{i}}\right) . \quad\left[M_{v_{i}}: \tilde{M}_{v_{i}}\right],\left[N_{v_{i}}: \tilde{N}_{v_{i}}\right]<$ $\infty$ yield $\sigma\left(\boldsymbol{Q}\left(v_{i} \otimes M_{v_{i}}\right)\right)=\boldsymbol{Q}\left(v_{i} \otimes N_{v_{i}}\right)$. This completes the proof of Theorem 4.

Q.E.D.

Theorem 2, 3, 4 yield Theorem at the begining of this paper.

$\S 4$.

In this section we give examples of positive lattices in Theorem.

Proposition. Let $L=Z\left[e_{1}, \cdots, e_{n}\right]$ be a quadratic lattice and put $c a_{i j}=B\left(e_{i}, e_{j}\right)$. Assume that

(0) $c, a_{i j} \in \boldsymbol{Q}, c>0$,

(1) $a_{i i}=1$ and $1-\sum_{j \neq i}\left|a_{i j}\right| \geq 0$ for $i=1, \cdots, n$, 
(2) for any non-empty subset $S$ of $\{1,2, \cdots, n\}$

$$
\#|S|-1 \geq \sum_{\substack{i, j \in S \\ i \neq j}}\left|a_{i j}\right| \text {. }
$$

Then $L$ is a positive lattice of E-type and $L=\tilde{L}$.

Proof. By scaling we may suppose $c=1$ without loss of generality. Let $M$ be a positive lattice with $m(M)=1$. Take any non-zero element $x=\sum_{i=1}^{n} e_{i} \otimes u_{i} \in L \otimes M$. Put $b_{i j}=a_{i j}|| a_{i j} \mid$ if $a_{i j} \neq 0$, =0 if $a_{i j}=0$, and $S=\left\{i \mid u_{i} \neq 0\right\}(\neq \phi)$. Then we have

$$
\begin{aligned}
Q(x) & =\sum a_{i j} B\left(u_{i}, u_{j}\right) \\
& =\sum Q\left(u_{i}\right)+\frac{1}{2} \sum_{i \neq j} a_{i j}\left(2 B\left(u_{i}, u_{j}\right)\right) \\
& =\sum Q\left(u_{i}\right)+\frac{1}{2} \sum_{i \neq j}\left|a_{i j}\right|\left(Q\left(b_{i j} u_{i}+u_{j}\right)-Q\left(u_{i}\right)-Q\left(u_{j}\right)\right) \\
& =\sum_{i \in S}\left(1-\sum_{\substack{j \in S \\
j \neq i}}\left|a_{i j}\right|\right) Q\left(u_{i}\right)+\frac{1}{2} \sum_{\substack{i, j \in S \\
i \neq j}}\left|a_{i j}\right| Q\left(b_{i j} u_{i}+u_{j}\right) \\
& \geq \#|S|-\sum_{\substack{i, j \in S \\
i \neq j}}\left|a_{i j}\right| \\
& \geq 1 .
\end{aligned}
$$

Hence $L$ is positive and $m(L) m(M) \geq m(L \otimes M) \geq 1 . \quad m(L) \leq 1, m(\mathbf{M})=1$ imply $m(L \otimes M)=1$ and $m(L)=1$. If $Q(x)=1$ and hence $x \in \mathfrak{M}(L \otimes M)$, then $b_{i j} u_{i}+u_{\jmath}=0$ and hence $u_{i}= \pm u_{j}$ for $i, j \in S$ with $i \neq j, a_{i j} \neq 0$. Suppose $S=S_{1} \cup S_{2}$ and $a_{i \jmath}=0$ if $i \in S_{1}, j \in S_{2}$; then $x=\left(\sum_{i \in S_{1}} e_{i} \otimes u_{i}\right)$ $+\left(\sum_{j \in S_{2}} e_{j} \otimes u_{j}\right)$ is an orthogonal sum and $x \in \mathfrak{M}(L \otimes M)$ implies that one of them must vanish. Thus we have $S_{1}$ or $S_{2}=\phi$ and then $u_{i}= \pm u_{j}$ for $i, j \in S$. Therefore $x$ should be $e \otimes u_{i}$ for $e \in L, i \in S$. By definition $L$ becomes a lattice of $E$-type and $m(L)=1$ implies $\mathfrak{M}(L) \supset\left\{e_{i}\right\}$ and hence $L=\tilde{L}$. Thus we complete the proof.

Q.E.D.

Remark. If $a_{i i}=1,\left|a_{i j}\right|<1 / n(i \neq j)$, then the conditions (1), (2) are satisfied and $\mathfrak{M}(L)=\left\{ \pm e_{i} \mid 1 \leq i \leq n\right\}$. In this case it is easy to see whether $L$ is indecomposable or not. Suppose that $L$ is indecomposable and $L=L_{1} \otimes L_{2}$. Then from our theorem follows that $L_{1}, L_{2}$ are of $E$-type and $\mathfrak{M}(L)=\mathfrak{M}\left(L_{1}\right) \otimes \mathfrak{M}\left(L_{2}\right), L_{i}=\tilde{L}_{i}$ and $\left|\mathfrak{M}\left(L_{i}\right)\right|=2 r k L_{i}$. Hence we can take minimal vectors as a basis of $L_{i}$, and then the matrix $\left(B\left(f_{i}, f_{j}\right)\right)$ corresponding to $L$, where $\left\{ \pm f_{i}\right\}=\left\{ \pm e_{i}\right\}$, is a tensor product of matrices corresponding to $L_{i}$ by their minimal vectors. Thus it is also easy to see whether $L$ is indecomposable with respect to tensor product or not. 


\section{REFERENCES}

[1] Y. Kitaoka, Scalar extension of quadratic lattices II, Nagoya Math. J., 67 (1977), 159-164.

[2] — - Tensor products of positive definite quadratic forms, Göttingen Nachr. Nr., 4 (1977).

[3] —- Tensor products of positive definite quadratic forms II, J. reine angew. Math., 299/300 (1978), 161-170.

[4] _ - Tensor products of positive definite quadratic forms IV, Nagoya Math. J., 73 (1979), 149-156.

[5] O. T. O'Meara, Introduction to quadratic forms, Berlin-Heidelberg-New York, 1963.

Department of Mathematics

Faculty of Science

Nagoya University

Chikusa-ku

Nagoya, 464, Japan 